SHORT REPORT

\title{
Hospital accessibility and infant death risk
}

\section{T J B Dummer, L Parker}

This study of all 4889 infant deaths within the cohort of all 287993 births in Cumbria, northwest England (1950-93), found no evidence of an increased risk of infant death with greater travel time to hospitals.

$\mathrm{R}$ isk of mortality from asthma increases with distance from hospital. ${ }^{1}$ In the developing world increasing distance from hospital has been shown to be a risk factor for perinatal mortality. ${ }^{2}$ With respect to the UK, Parker and colleagues ${ }^{3}$ found no evidence of an increased risk of stillbirth in relation to increasing distance from maternity hospitals in West Cumbria. Little is known about the risk of infant mortality in relation to hospital accessibility.

Accessibility to hospital can be defined in a number of ways: straight-line distance between the hospital and home; road travel distance; road travel time from home to hospital. In this study a geographical information system (GIS) was used to model road travel time to hospital. The aim of the study was to investigate whether geographical accessibility to hospitals affected the risk of infant mortality in Cumbria, northwest England, 1950-93. In addition, we investigated stillbirth risk in Cumbria in relation to hospital accessibility using a larger dataset than that considered by Parker and colleagues. $^{3}$

\section{METHODS}

This study used the Cumbrian Births Database (CBD), which comprises all 283668 live births, 4325 stillbirths and 4889 infant deaths in Cumbria, 1950-93 (described in Dummer and colleagues ${ }^{4}$ ). Postcode at birth, social class, birth order, multiple births, and year of birth were coded for the cohort and grid references of all births were derived from the postcode at birth. ${ }^{4}$ Infant deaths were coded into early neonatal (0-6 days old), neonatal (0-27 days old), and postneonatal (28 days to 1 year).

The grid reference and details of all hospitals in Cumbria open between 1950 and 1993 were captured in the GIS database (see fig 1). The road network and boundaries of built-up areas were also captured in the GIS. A travel time to hospital surface of isochrones-lines of equal travel time ${ }^{5}$ was generated by combining the road network, the location of built-up areas (which affects vehicle speeds on the roads), and the location of all hospitals. For the infant death analysis, general hospitals and hospitals with paediatric facilities were incorporated. For the stillbirth analysis all hospitals with a maternity facility were incorporated. Hospital locations included Lancaster Royal Infirmary, which is outside Cumbria but provides maternity and paediatric facilities for those in the south of the county. The grid reference of each birth was used to geographically locate individual births. Travel time to hospital, in relation to hospitals open in the year of birth, was derived for all individual live and still births.
Logistic regression was used to investigate the risk of infant mortality (early neonatal, neonatal, and postneonatal deaths) and stillbirth in relation to accessibility to hospital, adjusting for demographic risk factors (year of birth, social class, birth order, multiple births). Continuous and categorical odds ratios (ORs) with $95 \%$ confidence intervals (CIs) are presented. Significance was assessed through the likelihood ratio test statistic (LRTS) and inspection of the 95\% CIs. Because infant mortality fell dramatically over the study period $^{4}$ the analysis was stratified into four time periods: 1950-59, 1960-69, 1970-79, 1980-93. Hospital accessibility was defined by a function of travel time to hospital as (a) linear function in minutes, (b) categorical function grouping travel time into close, medium and far, using Conner's exponential grouping, ${ }^{6}$ resulting in: category (1) 0-17 minutes; category (2) >17-35 minutes; category (3) >35 minutes.

\section{RESULTS}

Results are presented in table 1 . The median travel time to hospital was 12 minutes; maximum 70 minutes, minimum $<$ l minute. Risk of infant death did not increase with increasing travel time to hospital, either overall or within time periods. In most cases continuous ORs were very close to 1.00, indicating little variation in risk of infant death in relation to proximity to hospitals. During 1960-69 the OR for risk of postneonatal death was higher in the furthest travel time category compared to the nearest category. While the 95\% CI indicated a significantly increased OR, the $p$ value was of borderline significance only $(p=0.05)$. By contrast the continuous OR showed no significant variation in risk for postneonatal deaths with proximity to hospital in 1960-69. The significant increasing risk of postneonatal death in the travel time category furthest from hospital in 1960-69 was only apparent after adjustment for demographic risk factors and is therefore likely to be a chance finding. The infant death analysis was repeated incorporating maternity facilities in the travel time function, but this did not materially affect the results. There was no evidence of increasing risk of stillbirth with increasing travel time to hospital (results not presented), confirming the results in Parker and colleagues. ${ }^{3}$

\section{DISCUSSION AND CONCLUSIONS}

Relying on straight-line distance as an indicator of geographical accessibility to hospital facilities is a crude approximation of geographical accessibility-accessibility depends on physical distances and transport times, which depend on factors including road types and vehicle speeds. This study utilised GIS to estimate road travel time to hospitals; potentially a more accurate assessment of geographical accessibility. $^{5}$

There was no evidence to suggest that living further from hospitals, in terms of road travel time, increased the risk of infant death or stillbirth in Cumbria. A limitation of this study was lack of data after 1993, which are more relevant to hospital location planning. Therefore, although we found no variation in infant death or stillbirth risk with increasing travel time to hospital, this evidence cannot be used to justify 


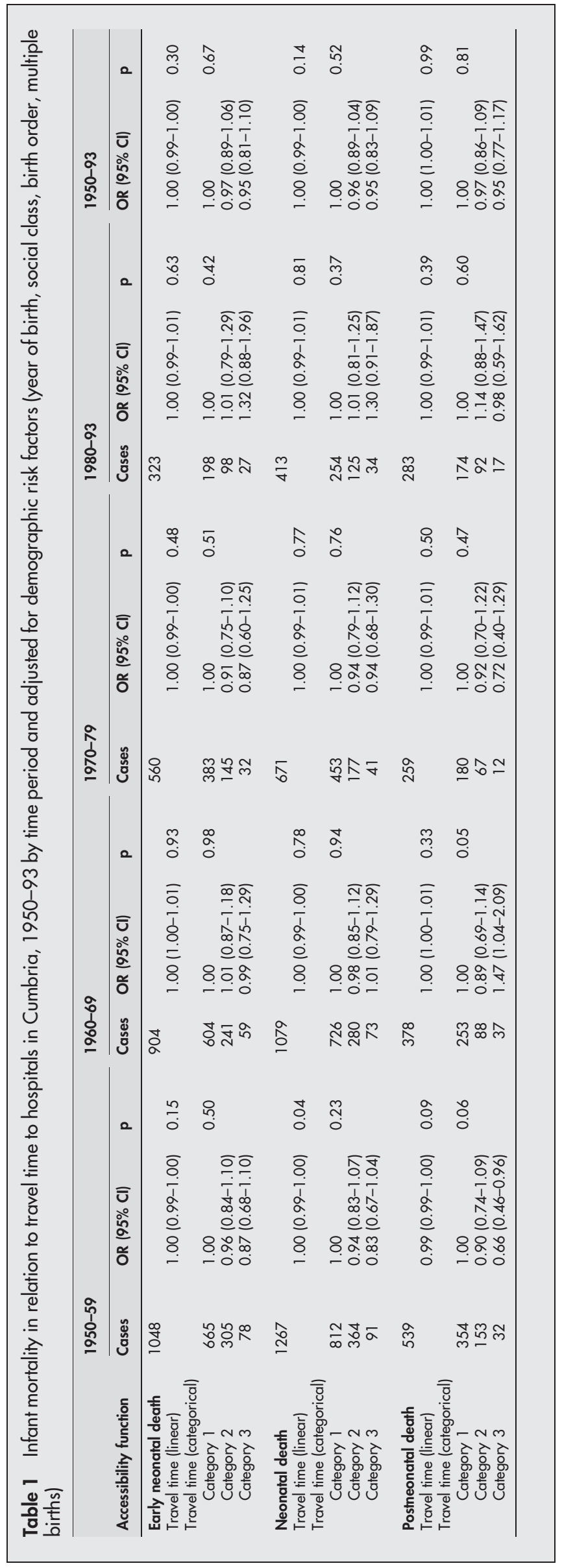




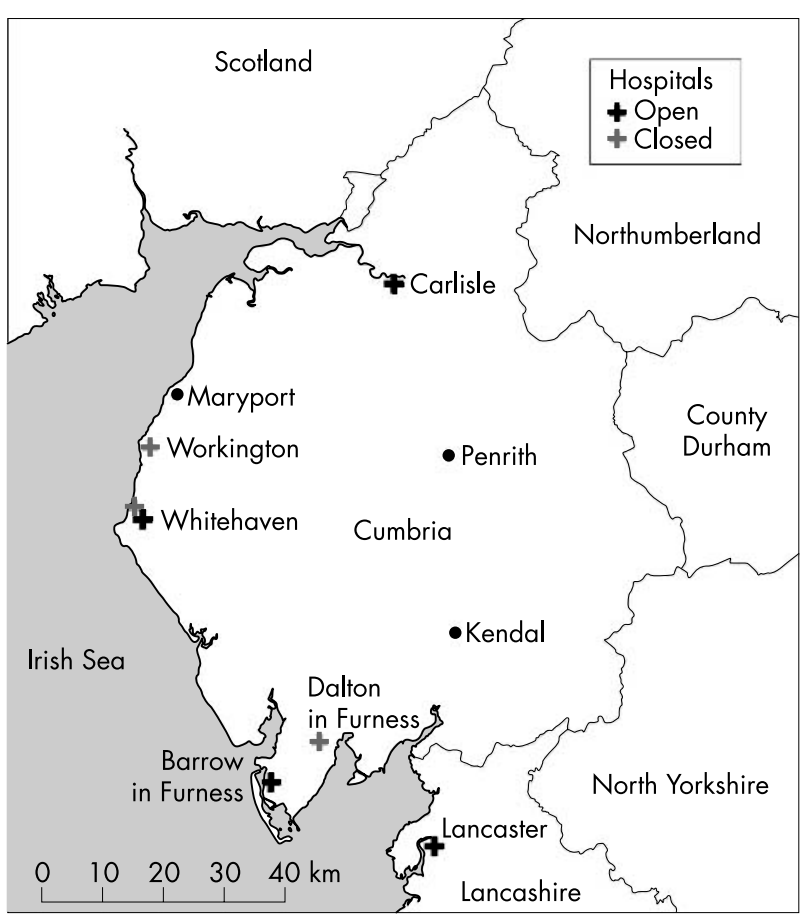

Figure 1 Location of hospitals in Cumbria, 1950-93.

further centralisation of hospital services. However, the data may be useful to support siting of future paediatric hospital facilities.

\section{ACKNOWLEDGEMENTS}

This study used Bartholomew digital data which is copyright of Bartholomew Ltd and used with permission.

\section{Authors' affiliations}

T J B Dummer, School of Social Science, Liverpool John Moores University, Henry Cotton Campus, 15-21 Webster Street, Liverpool L3 2ET, UK

L Parker, Paediatric and Lifecourse Epidemiology Group, School of Clinical Medical Sciences, University of Newcastle, Sir James Spence Institute of Child Health, Royal Victoria Infirmary, Newcastle NE1 4LP, UK

Correspondence to: Dr T Dummer, School of Social Science, Liverpool John Moores University, Henry Cotton Campus, 15-21 Webster Street, Liverpool L3 2ET, UK; t.dummer@livjm.ac.uk

Accepted 21 June 2003

\section{REFERENCES}

1 Jones AP, Bentham G, Horwell C. Health service accessibility and deaths from asthma. Int J Epidemiol 1999;28:101-5.

2 Fikree FF, Midhet F, Sadruddin S, et al. Maternal mortality in different Pakistani sites: ratios, clinical causes and determinants. Acta Obstet Gynecol Scand 1997;76:637-45.

3 Parker L, Dickinson HO, Morton-Jones T. Proximity to maternity services and stillbirth risk. Arch Dis Child Fetal Neonatal Ed 2000;82:F167-8.

4 Dummer TJB, Dickinson HO, Pearce MS, et al. Stillbirth risk with social class and deprivation: no evidence for increasing inequality. J Clin Epidemiol 2000;53:147-55.

5 O'Sullivan D, Morrison A, Shearer J. Using desktop GIS for the investigation of accessibility by public transport: an isochrone approach. Int J Geog Info Sci 2000;14:85-104.

6 Connor RJ. Grouping of testing trends in categorical data. J Am Stat Assoc 1972;67:601-604. 